\title{
Evaluation of Liver Stiffness using Magnetic Resonance Elastography in Hepatic Steatosis
}

Dr. Nikhith Soman ${ }^{1 *}$, Dr. Amit Kharat ${ }^{1}$, Dr. Jacob Jesson ${ }^{1}$

${ }^{1}$ DY Patil Medical College, Pune, India

DOI: $10.36347 /$ sjams.2021.v09i03.033

| Received: 06.03.2021 | Accepted: 24.03.2021 | Published: 28.03.2021

*Corresponding author: Dr. Nikhith Soman

Liver fibrosis is a predominantly asymptomatic disease, picking up early changes in stiffness of liver is crucial for detecting the same. Hepatic steatosis as such cannot cause fibrosis but in the setting of repeated steatohepatitis there is an increased risk. In this study we try to assess the changes in liver stiffness in patients with fatty liver and find out any correlation between percentage of fat and stiffness. 50 candidates were selected with fatty liver changes on ultrasonography and were made to undergo Magnetic Resonance Elastography (MRE). When liver fat percentage was compared to liver stiffness, no statistically significant correlation was found with $p$ value $>0.05$. However, only eight candidates had normal stiffness, 18 had mild increase in stiffness (stage F0), others had significant increase in stiffness. MRE can however play a crucial role as a non-invasive technique in the early detection of fibrosis before it reaches a more advanced irreversible stage.

Keywords: Liver fibrosis, liver stiffness, predominantly asymptomatic disease, Hepatic Steatosis.

Copyright $\left({ }_{0} 2021\right.$ The Author(s): This is an open-access article distributed under the terms of the Creative Commons Attribution 4.0 International License (CC BY-NC 4.0) which permits unrestricted use, distribution, and reproduction in any medium for non-commercial use provided the original author and source are credited.

\section{INTRODUCTION}

Fatty liver or hepatic steatosis is a common finding during abdominal ultrasonography, however it is often ignored. Simple steatosis as such is benign and is not seen to cause any major health issue. However, once the vicious cycle of steatohepatitis sets in, the consequent risk of fibrosis and cirrhosis increases. The main problem is that the bulk of these processes preceding the development of cirrhosis is silent, hence the goal is always to pick up the early changes of fibrosis which can be reversible [1].

Fibrosis is associated with increased stiffness of the liver, so detecting changes to liver stiffness as it begins and trying to prevent progression is crucial. Many techniques exists to assess liver stiffness, the most advanced, non invasive and comprehensive approach is using Magnetic Resonance Elastography (MRE) [2].

The basic principle of MRE is based on the principle that there is an increase in velocity of mechanical waves as it travels through increasing stiffness mediums, in other words waves travel fastest through harder mediums. These propagating waves are captured using motion encoded MRI sequences and stiffness is calculated [2].

\section{Materials And Methods}

The prospective study was conducted in the tertiary care center over a period of 2 years after obtaining clearance from the ethical committee. A total of 50 patients underwent the study. First patients are screened with the help of ultrasonography for fatty changes of liver and graded based on liver echogenicity in relation to the echogenicity of portal vessel walls and diaphragm

The patients were then subjected to MR Elastography (Siemens Magnetom Vida 3 Tesla) evaluation and the liver Elastograms are developed. The quantification of liver fat using MRI (Siemens Liver lab) was also done. The correlation with liver fat and liver stiffness was done along with various other patient-specific characteristics.

MRE requires additional hardware apart from the body coils and specific sequences. This machine consists of an active driver outside the magnet room, producing regular vibrations of low frequency. These vibrations are conveyed by a flexible tube to a drumlike, passive conductor, which is directly positioned over the liver and held up by the abdominal binder against the anterior right chest wall. A phase-difference 
Nikhith Soman et al; Sch J App Med Sci, Mar, 2021; 9(3): 462-467

gradient-echo sequence captures the propagating shear waves [3].

The patient is introduced in a supine position. The passive driver is mounted in the midclavicular line above the lower right chest wall at the level of xiphisternum,in direct contact with the body wall; the passive driver is secured with an elastic band. The passive driver is connected through a plastic tube to the active driver outside the scanning room [15].

2D gradient-echo sequences with cyclic motion-encoding gradients (MEG) were used for MR Elastography.at quantification was done using - T1 VIBE-eDIXON, T1 VIBE-qDIXON and HISTO sequences.

\section{Data Collection Method and Statistical Analysis}

Data has been collected from the subjects on a pretested proforma. Continuous variables like liver stiffness, liver fat fraction were expressed as mean (standard deviation). The distribution of categorical variables like age and gender, were summarized as proportions. The association of liver stiffness and fat fraction, were assessed using a correlation coefficient. A $p$ value of less than 0.05 was considered as statistically significant.

\section{RESULTS}

Out of the 50 patients selected for the study based on the qualitative assessment on ultrasonography, 20 had grade 1 fatty liver, 18 had grade 2 fatty liver and 12 had grade 3 fatty liver.

The ultrasound grading grading was mostly comparable to that of quantitative fat assessment done on MRI. The mean fat percentage compared to their ultrasound grades was corresponding with statistical significance.

When liver fat percentage was compared to liver stiffness, no statistically significant correlation was found with $\mathrm{p}$ value $>0.05$. No linear correlation could be established. However, only eight candidates had normal stiffness, 18 had mild increase in stiffness (stage F0), other had significant increase in stiffness but was not in correlation with liver fat percentage.

Table-1: USG fatty liver gradings and their corresponding MRI fat fractions

\begin{tabular}{|c|c|c|c|c|}
\hline & $<7 \%$ & 7 to $22 \%$ & $<22$ to $33 \%$ & $>33 \%$ \\
\hline USG Grade 1 & 6 & 14 & 0 & 0 \\
\hline USG Grade 2 & 0 & 14 & 4 & 4 \\
\hline USG Grade 3 & 0 & 0 & 8 & 4 \\
\hline
\end{tabular}

Table-2: Means of fat fractions in USG fatty liver grades

\begin{tabular}{|c|c|c|}
\hline USG grade & Mean of fat percentage on MRI & SD \\
\hline I & 9.31 & 4.7673 \\
\hline II & 19.2667 & 5.444 \\
\hline III & 30.3333 & 6.0503 \\
\hline \multicolumn{2}{|c|}{ The $p$-value is <.00001. The result is significant. } \\
\hline
\end{tabular}

Table-3: Correlation of fat fraction with liver stiffness

\begin{tabular}{|c|c|c|c|c|c|c|}
\hline & Normal & F0 & F1 & F2 & F3 & F4 \\
\hline Normal & 4 & 0 & 0 & 2 & 0 & 0 \\
\hline MRI Grade 1 & 4 & 16 & 0 & 0 & 4 & 4 \\
\hline MRI Grade 2 & 2 & 2 & 0 & 4 & 4 & 0 \\
\hline MRI Grade 3 & 0 & 0 & 4 & 0 & 0 & 0 \\
\hline \multicolumn{7}{|c|}{ P $=0.5288$, Not significant } \\
\hline
\end{tabular}




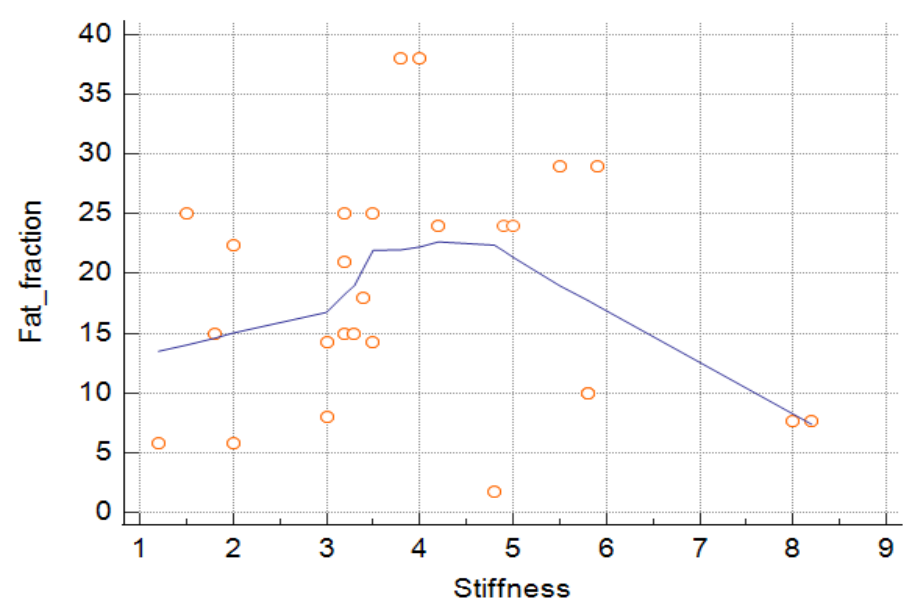

Image no 1: Scatter diagram showing no linear correlation with fat fraction and liver stiffness

\section{DISCUSSION}

Quantitative evaluation of the liver using MRI, like MRE and chemical shift imaging is becoming a highly useful method to noninvasively assess tissue characteristics, hence, providing a holistic approach to liver health. However, interrelationships between different tissue characteristics are not well understood, for the correct analysis of the quantitative MRI findings we require better understanding of these relationships [3]. The main focus of the study was to assess the role of MRE of liver in evaluating changes in liver stiffness with varying degrees of hepatic steatosis. Fatty liver or hepatic steatosis is a wide spread and mostly, asymptomatic condition often ignored in clinical practice. However, it is sometimes the only indicator before the onset of fibrosis. All patients with steatosis don't always end up with fibrosis or cirrhosis. It depends on various other patient specific and environmental factors [3]. Hence in this research, we investigated the ties in a small cohort of patients with fatty liver changes on ultrasonographic studies, the relation between tissue characteristics derived from MRI like that of liver fat and liver stiffness.

Since we selected patients with fatty changes on USG (based on liver echogenicity), we tried comparing the consistency of ultrasound findings with the fat fraction observed on MRI, later is a more quantitative estimate using the principle of chemical shift imaging. We will try to discuss the various findings under the following headings of liver fat and liver stiffness, describing significant relationships.

\section{Liver Stiffness}

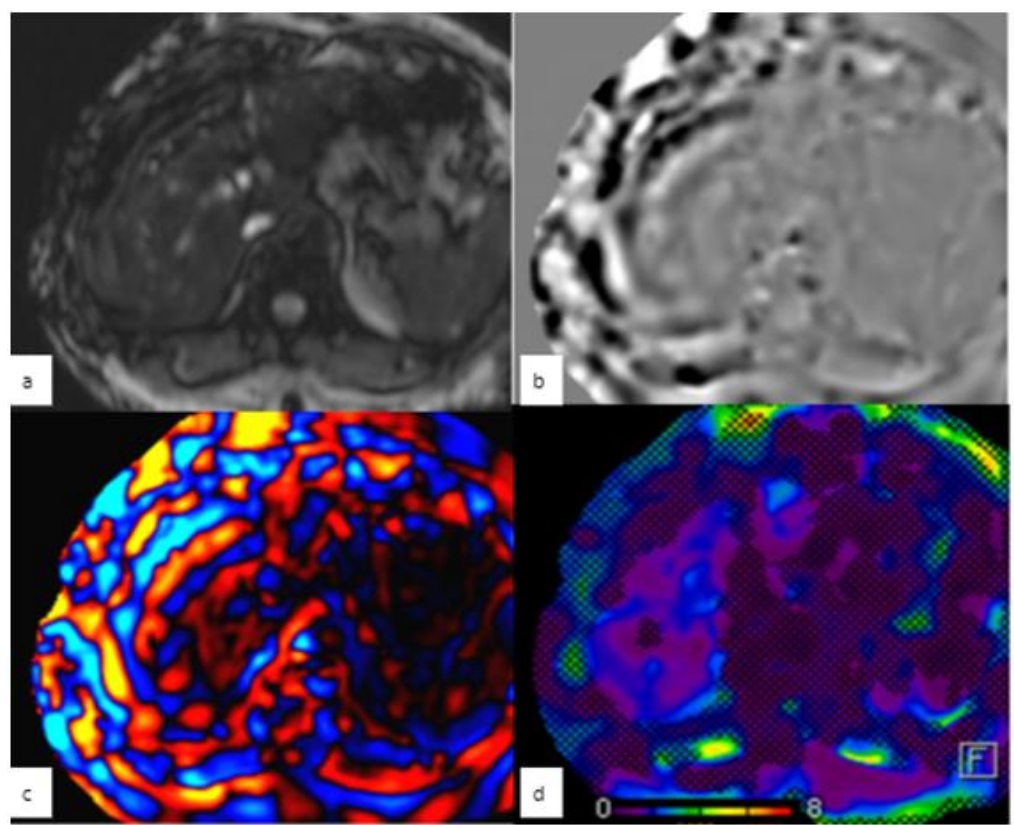

Image no. 2: These are magnitude (a), phase (b), wave (c) and elastogram (d) images obtained using the 3T Siemens vida platform showing normal liver with mean stiffness measurement of $1.2 \mathrm{kpa}$ 
The liver stiffness was calculated from the Elastograms after taking the average of the ROIs and then graded in relation to the METAVIR classification for fibrosis, we followed the Yin at el study to grade our patients [3]

In our study no correlation could be demonstrated between liver fat and liver stiffness, there were many cases where there was a very high level of fat percentage, while the stiffness was towards the normal end, vice versa there were also cases with increased stiffness of liver without any significant fat deposit. The latter agrees with the theory of fat replacement with fibrous tissue in the late stages of fibrosis. However, the relationship between fat and stiffness in liver tissue has been controversial with many earlier studies establishing a linear relationship and newer studies with MRI techniques stating no direct relationship, like that of our study [4-6].

A study by Yin et al., to detect fibrosis in the liver using magnetic resonance Elastography also agreed with ours, in which they also assessed any relation between liver fat and stiffness. They concluded that liver fibrosis was independent of liver fat [3]
Chen et al., also did similar study in nonalcoholic hepatosteatosis using MRE, they also concluded the same, observing that no significant increase in stiffness was noted in simple steatosis. They also found that in advanced cases of fibrosis the fat percentage was very low [4].

An interesting study done recently showed that, in obese patients when liver stiffness was compared before and after weight loss, there was considerable decrease in stiffness. However this study did not assess the exact fat percentage of the liver for comparison, rather under the assumption that fat fraction would have reduced in weight loss. This could also be attributed to the decrease in inflammatory processes in the liver; many studies have shown significant increase in liver stiffness in cases of steatohepatitis [7].

Earlier studies using other modalities of Elastography, based on ultrasound techniques showed contradicting results, where the stiffness increased with increasing degree of steatosis, this is likely due overestimation of stiffness $[8,9]$.

\section{Liver Fat}

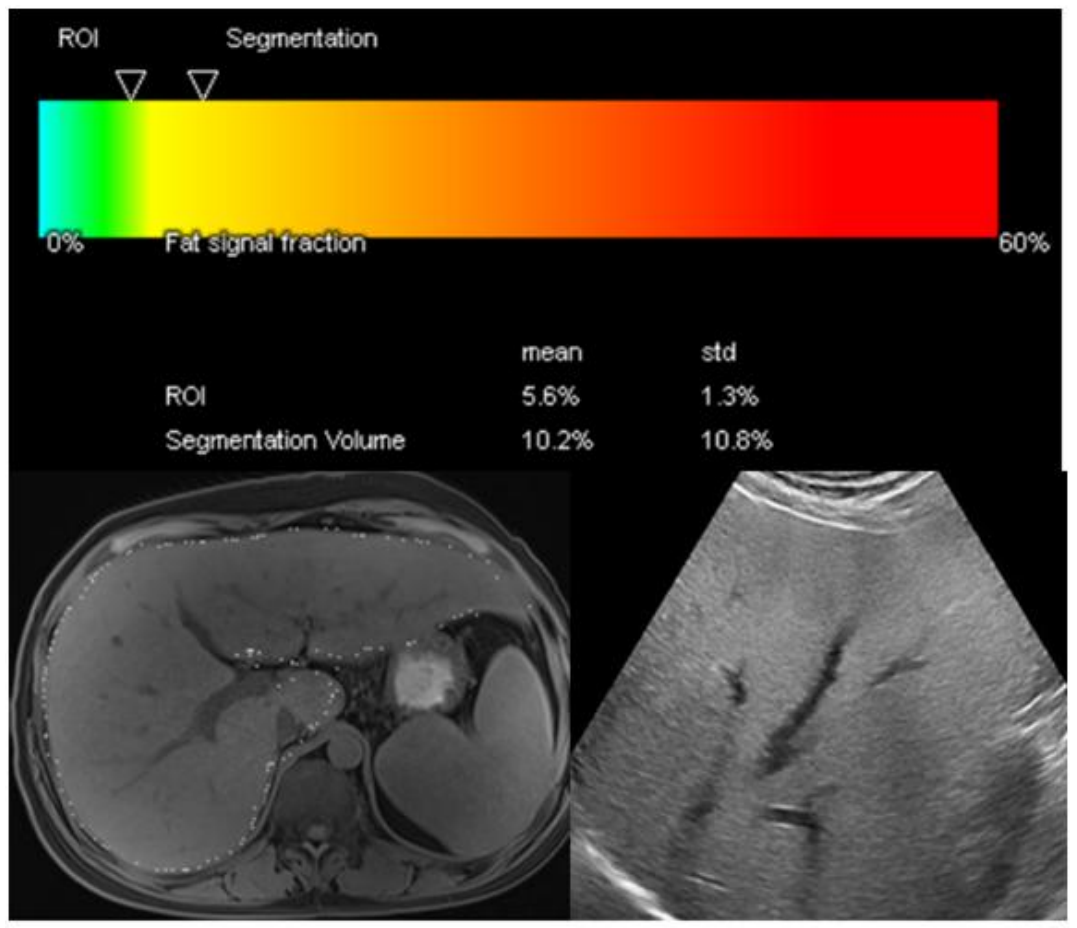

Image no.3: The image shows fat quantification done on a 3 Tesla MRI with automatic segmentation of the whole liver and quantifying the fat percentage in the liver along with its ultrasound image. In this patient the liver was analysed and showed a total of $10.2 \%$ fat

Liver fat was first qualitatively assessed on USG and was compared with the fat fraction estimated on MRI. Out of the 50, all had fatty changes on USG with majority having grade I changes (20 patients).
The fat in the liver was quantified on MRI and represented as fat fractions, a large portion of the patients showed grade 1 fatty changes (28 patients), there were even few which showed normal fat fraction in the liver ( 6 patients). The mean fat fraction in each 
Nikhith Soman et al; Sch J App Med Sci, Mar, 2021; 9(3): 462-467

patient showed proportional increase according to the grading on USG. There was also a positive correlation with the USG grading and the MRI fat fraction noted in the correlation tests. There were some degrees of over diagnosis on USG with many cases showing normal fat fraction on MRI. There were also some differences noted in the USG and MRI liver fat classification systems, the patients' grades on USG mostly had fat percentage belonging to a lower grade on MRI based grading. These differences were expected as the USG based grading is operator dependent and varied according to the machines and its settings.

M. L. Kromrey et al., study also agrees with our study showing high comparability of USG and MRI liver fat assessment, they also showed that the sensitivity increased with increasing quantity of fat [10].

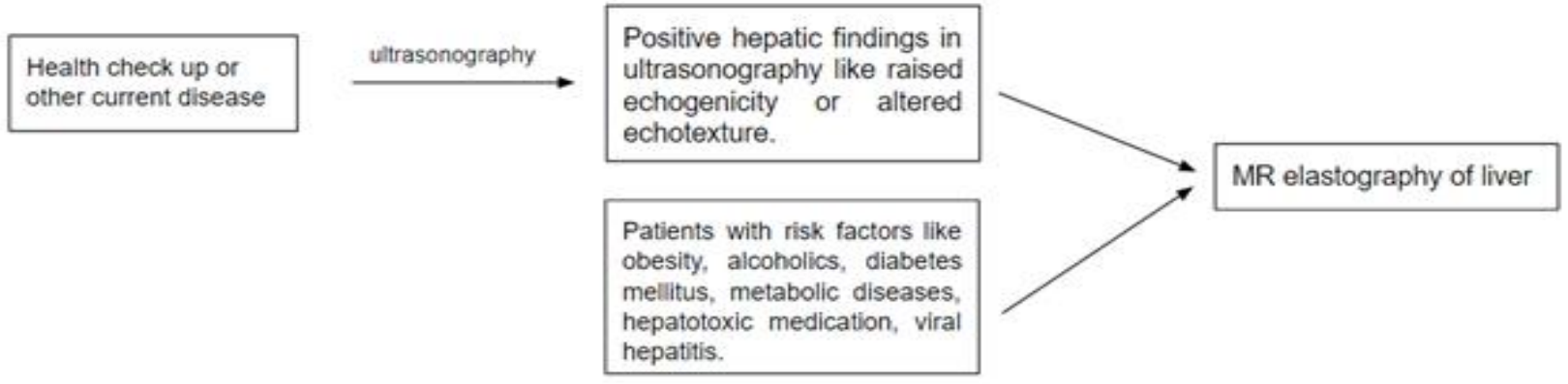

Image no. 4: Suggested patient workflow for indicating Magnetic Resonance Elastography

\section{LIMITATIONS}

This study had few limitations. First, the sample size is small, thus limits the generalization of the results to the population in large. Second, biopsy correlation was not available, the grades of fibrosis were judged based on cutoffs of other studies with histopathological correlation. Third, other clinical and laboratory parameters were not compared with the liver stiffness and fat fractions, other factors like ethnicity, race were not correlated. Fourth, the duration of risk factors and onset of liver fibrosis was not evaluated. Many of the variables could have had an impact on the disease based on the duration.

\section{CONCLUSION}

In this study we mainly tried to analyze any relationship between liver fat and liver stiffness. The patients were selected on the basis of fatty changes on ultrasonography and later fat was also quantified using MRI based techniques. This data was then directly correlated with the liver stiffness in kilopascals using MRE. The statistical analysis showed no linear relationship between the same. It was also observed that many times when patients with very high percentages of fat showed relatively lower levels of stiffness. Thus, simple steatosis alone cannot lead to fibrotic changes in the liver; it needs to stimulate a state of chronic inflammation entering the stage of steatohepatitis. This step is often dependent on various patient specific environmental and genetic factors [11-13].

Magnetic resonance Elastography is a relatively new technique that assesses the mechanical properties of tissues in a non-invasive manner. It is most effectively used in evaluation of liver stiffness. It can play a crucial role in the early detection of fibrosis before it reaches a more advanced irreversible stage. It becomes more important in case of chronic liver disease as its course is largely asymptomatic until it reaches liver failure or cirrhosis. Thus, incorporating this into normal routine can prove very useful. One of the only easily appreciated predictors of liver health is fatty changes on ultrasonography, which is sometimes the only finding noted before the stage of cirrhosis $[14,15]$.

\section{REFERENCES}

1. Asrani SK, Devarbhavi H, Eaton J, Kamath PS. Burden of liver diseases in the world. Journal of hepatology. 2019 Jan 1;70(1):151-71.

2. Mariappan YK, Glaser KJ, Ehman RL. Magnetic resonance elastography: a review. Clinical anatomy. $2010 \mathrm{Jul} ; 23(5): 497-511$.

3. Yin M, Talwalkar JA, Glaser KJ, Manduca A, Grimm RC, Rossman PJ, Fidler JL, Ehman RL. Assessment of hepatic fibrosis with magnetic resonance elastography. Clinical Gastroenterology and Hepatology. 2007 Oct 1;5(10):1207-13.

4. Jun Chen, Jayant A. Talwalkar, Meng Yin, Kevin J. Glaser, Schuyler O. Sanderson, Richard L. EhmanEarly Detection of Nonalcoholic Steatohepatitis in Patients with Nonalcoholic Fatty Liver Disease by Using MR Elastography

5. Loomba R, Wolfson T, Ang B, Hooker J, Behling C, Peterson M, Valasek M, Lin G, Brenner D, Gamst A, Ehman R. Magnetic resonance elastography predicts advanced fibrosis in patients with nonalcoholic fatty liver disease: a prospective study. Hepatology. 2014 Dec;60(6):1920-8.

6. Joshi M, Dillman JR, Singh K, Serai SD, Towbin AJ, Xanthakos S, Zhang B, Su W, Trout AT. Quantitative MRI of fatty liver disease in a large 
Nikhith Soman et al; Sch J App Med Sci, Mar, 2021; 9(3): 462-467

pediatric cohort: correlation between liver fat fraction, stiffness, volume, and patient-specific factors. Abdominal Radiology. 2018 May 1;43(5):1168-79.

7. Patel NS, Hooker J, Gonzalez M, Bhatt A, Nguyen P, Ramirez K, Richards L, Rizo E, Hernandez C, Kisseleva T, Schnabl B. Weight loss decreases magnetic resonance elastography estimated liver stiffness in nonalcoholic fatty liver disease. Clinical gastroenterology and hepatology: the official clinical practice journal of the American Gastroenterological Association. 2017 Mar;15(3):463.

8. Petta S, Maida M, Macaluso FS, Di Marco V, Camma C, Cabibi D, Craxì A. The severity of steatosis influences liver stiffness measurement in patients with nonalcoholic fatty liver disease. Hepatology. 2015 Oct;62(4):1101-10.

9. Conti F, Vukotic R, Foschi FG, Domenicali M, Giacomoni P, Savini S, Lanzi A, Dall'Aglio AC, Saini G, Matroroberto M, Bernardi M. Transient elastography in healthy subjects and factors influencing liver stiffness in non-alcoholic fatty liver disease: An Italian community-based population study. Digestive and Liver Disease. 2016 Nov 1;48(11):1357-63.

10. Kromrey ML, Ittermann T, Berning M, Kolb C, Hoffmann RT, Lerch MM, Völzke H, Kühn JP. Accuracy of ultrasonography in the assessment of liver fat compared with MRI. Clinical radiology. 2019 Jul 1;74(7):539-46.

11. Venkatesh SK, Yin M, Takahashi N, Glockner JF, Talwalkar JA, Ehman RL. Non-invasive detection of liver fibrosis: MR imaging features vs. MR elastography. Abdominal imaging. 2015 Apr 1;40(4):766-75.

12. SrinivasaBabu A, Wells ML, Teytelboym OM, Mackey JE, Miller FH, Yeh BM, Ehman RL, Venkatesh SK. Elastography in chronic liver disease: modalities, techniques, limitations, and future directions. Radiographics. 2016 Nov;36(7):1987-2006.

13. Venkatesh SK, Yin M, Ehman RL. Magnetic resonance elastography of liver: technique, analysis, and clinical applications. Journal of magnetic resonance imaging. 2013 Mar;37(3):54455.

14. Singh S, Venkatesh SK, Wang Z, Miller FH, Motosugi U, Low RN, Hassanein T, Asbach P, Godfrey EM, Yin M, Chen J. Diagnostic performance of magnetic resonance elastography in staging liver fibrosis: a systematic review and meta-analysis of individual participant data. Clinical Gastroenterology and Hepatology. 2015 Mar 1;13(3):440-51.

15. Venkatesh SK, Wang G, Lim SG, Wee A. Magnetic resonance elastography for the detection and staging of liver fibrosis in chronic hepatitis B. European radiology. 2014 Jan;24(1):70-8. 